\title{
Explicaciones de los estudiantes de grado quinto al resolver problemas relacionados con progresiones aritméticas ${ }^{1}$
}

\author{
Mobilization represent mathematical competence from a \\ didactic proposal by studying the linear function \\ Mobilização representar competência matemática a partir \\ de uma proposta didática, estudando a função linear
}

Recibido: mayo de 2013

Aceptado: agosto de 2013
Cindy Catalina Mendoza Bedoya ${ }^{2}$

Janeth Hurtado Betancur ${ }^{3}$

Jorge Enrique Mercado Romero ${ }^{4}$

\section{Resumen}

En la práctica pedagógica se adelanta una investigación en la cual se analizan las explicaciones escritas o verbales en estudiantes de grado quinto al resolver problemas relacionados con progresiones aritméticas.

La investigación tiene como antecedentes las observaciones de clases, el rastreo de textos escolares colombianos y pruebas saber; adicionalmente las lecturas de: los Lineamientos Curriculares de Matemáticas (1998), los Estándares Básicos de Competencias (2003), y algunas investigaciones relacionadas con el estudio de patrones y regularidades a nivel nacional e internacional. (Merino, 2012; Castro, E, Cañadas, M. C. \& Molina, M., 2010)

Se emplea una metodología cualitativa, considerando los casos particulares en su contexto natural. Es por esto que se aplican los instrumentos para analizar la información y proponer conclusiones.

Palabras clave: Explicaciones; patrones; progresiones aritméticas; aprendizaje; procesos cognitivos; razonamiento inductivo; otras nociones de Educación Matemática; resolución de problemas; resolución y estrategias.

\footnotetext{
Abstract

pedagogical practice conducting an investigation in which we analyze the written or oral explanations in fifth grade students to solve problems related to arithmetic progressions.

The background research is classroom observations, tracking and testing textbooks Colombians know; further readings: Mathematics Curriculum Guidelines (1998), Basic Skills Standards (2003), and some research
}

1 Artículo de Investigación. Este trabajo es asesorado por el profesor John Henry Durango Urrego, Magíster en Educación Matemática, Universidad de Antioquia. Integrante del Grupo de Investigación en Educación Matemática e Historia - EDUMATH, Universidad de Antioquia-Universidad Eafit.

2 Universidad de Antioquia. Medellin, Colombia. Contacto: catika_07@hotmail.com

3 Universidad de Antioquia. Medellin, Colombia. Contacto: janeth.hurtado@hotmail.com to:

4 Universidad de Antioquia. Medellin, Colombia. Contacto: kandu57@hotmail.com 
related to the study patterns and regularities nationally and internationally. (Merino, 2012; Castro, E, Cañadas, MC \& Molina, M., 2010)

It employs a qualitative methodology, considering the particular cases in their natural context. That's why the tools are applied to analyze information and propose conclusions.

Keywords: Explanations, patterns, arithmetic progressions, learning cognitive processes inductive reasoning, other notions of mathematics education, problem solving, resolution and strategies.

\section{Resumo}

A prática pedagógica a realização de uma investigação na qual analisamos as explicações orais ou escritas em estudantes do ensino fundamental para resolver problemas relacionados com progressões aritméticas.

A pesquisa de fundo são as observações em sala de aula, acompanhamento e testes manuais colombianos sabem; novas leituras: Matemática Diretrizes Curriculares (1998), Competências padrões básicos (2003), e algumas pesquisas relacionadas ao estudo padrões e regularidades nacional e internacional. (Merino, 2012; Castro, E, Cañadas, MC \& Molina, M., 2010)

Ele emprega uma metodologia qualitativa, considerando os casos particulares em seu contexto natural. É por isso que as ferramentas são aplicadas para analisar as informações e propor conclusões.

Palavras-chave: Explicações, padrões, progressões aritméticas, a aprendizagem de processos cognitivos raciocínio indutivo, outras noções de educação matemática, resolução de problemas, resolução e estratégias.

\section{Presentación del problema}

Esta investigación se realiza entre los años 2012 y 2013 por estudiantes de la Universidad de Antioquia, pertenecientes al programa "Licenciatura en Educación Básica con Énfasis en Matemáticas”, cuya práctica pedagógica permitió detectar e intervenir una problemática particular en relación con la clase de matemáticas de la Institución Educativa Bello Horizonte. En esta medida, las causas que originaron la presente problemática son en primer lugar, la fragmentación entre los temas abordados en clase y las pruebas saber; en segundo lugar, existe inconsistencia entre las pruebas de período académico y el proceso de enseñanza; en tercer lugar, no hay concordancia entre la malla curricular y el desarrollo de las clases; y finalmente, se presentan contradicciones entre teoría y los ejercicios propuestos en algunos textos escolares con respecto al tema de secuencias, especialmente la predicción de patrones y regularidades.

Es por esto que surgió la necesidad de conocer las disposiciones del Ministerio de Educación Nacional en referencia al razonamiento inductivo y las progresiones aritméticas plasmadas en los Lineamientos Curriculares de Matemáticas (MEN, 1998) y los Estándares Básicos de Competencias (MEN, 2003), encontrando en éstos que, el razonamiento se asume como un proceso de generalización, sin presentar una definición explícita sobre las progresiones aritméticas. Además, se realizó una búsqueda de investigaciones y referentes en Colombia relacionados con el razonamiento inductivo, las progresiones aritméticas, los patrones y las regularidades, evidenciando que poco se han abordado dichos temas en relación con el grado de escolaridad e interés de esta investigación. En cuanto al contexto internacional, la tesis Doctoral 
de Cañadas (2007) y la tesis de Maestría de Merino (2012) fueron insumos para el presente trabajo. Los siguientes antecedentes apoyaron y direccionaron la elección de la pregunta de investigación.

El proceso de observación e intervención de práctica se desarrolló en los grados cuarto y quinto de básica primaria de una institución de carácter oficial, cuyos grupos se caracterizaban por tener respectivamente 35 y 40 estudiantes, con edades que oscilan entre los 8 y 11 años, y que pertenecen a los estratos socioeconómicos 1 y 2 . Por tanto, el problema de investigación se enuncia en los siguientes términos: ¿Cómo explican los estudiantes de grado quinto, problemas relacionados con progresiones aritméticas? Para dar respuesta a esta pregunta, se formuló el siguiente objetivo: analizar el contenido de las explicaciones escritas o verbales que manifiestan los estudiantes de grado quinto al resolver problemas relacionados con progresiones aritméticas.

Figura 1

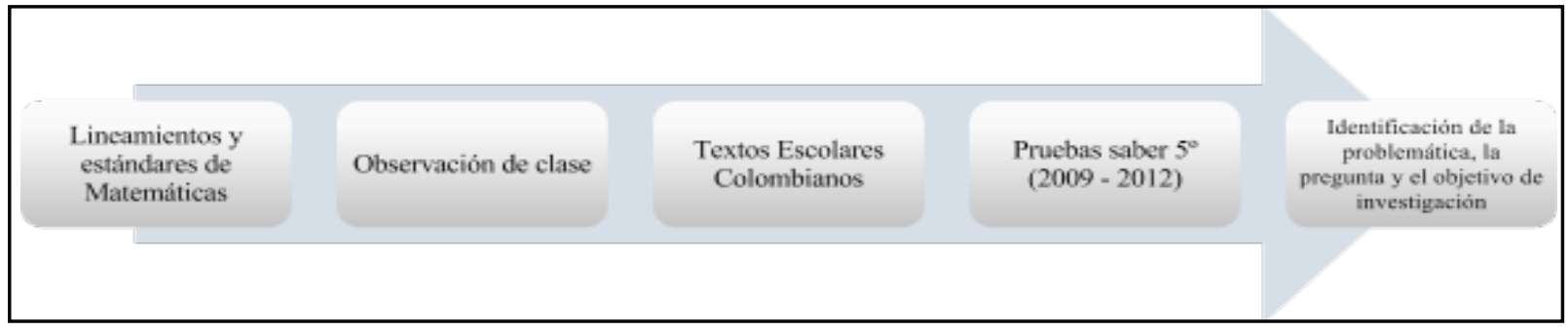

Fuente: Elaboración propia

\section{Marco de referencia conceptual}

El presente trabajo de investigación tiene como eje central la definición de Balacheff (2000) acerca del razonamiento, el cual plantea que "es la actividad intelectual no completamente explícita que se ocupa de la manipulación de la información dada o adquirida, para producir una nueva información" (p. 13). Ahora bien, en correspondencia con Balacheff, le asignaremos el término procesos de validación a esta misma actividad cuando tenga como fin asegurarse de la validez de una proposición y, eventualmente, producir una explicación, una prueba o una demostración.

Es de precisar, que el razonamiento que se explora en el presente trabajo es el inductivo, se ha tenido en cuenta la definición de Pólya (1945) con respecto a esta temática, ya que se aborda desde la resolución de problemas, planteando que éste requiere del trabajo con casos particulares, de la búsqueda de patrones basados en la regularidad observada en los casos particulares, de la formulación de una conjetura de acuerdo con el patrón, y de la comprobación posterior de dicha conjetura.
En esta misma línea, Castro, Cañadas \& Molina (2010) recoge algunos planteamientos y definiciones en relación con el razonamiento inductivo entre los que se destaca el de Pólya, quién plantea que el razonamiento inductivo es el razonamiento natural, que da lugar al conocimiento científico, mediante la obtención de leyes generales a partir de la observación de casos particulares. De igual modo, Merino (2012) establece que tanto la observación de casos particulares como la abstracción, permiten llegar a la generalización mediante el reconocimiento de patrones y regularidades de lo que es común.

Desde Pólya (1966), Mason (1999) y Gobernación de Antioquia (2005) se puede constatar que el reconocimiento de patrones y regularidades conducen a la generalización. Es así como Pólya señala que la habilidad para generalizar se lleva a cabo mediante un proceso de reconocimiento de patrones y regularidades; aunado a lo anterior, Mason (1999) establece que la identificación de patrones y regularidades en el ser humano llega desde antes de ingresar a la escuela y de igual modo, Gobernación de Antioquia (2005) afirma que: 
Un patrón es una propiedad, una regularidad, una cualidad invariante que expresa una relación estructural entre los elementos de una determinada configuración, disposición, composición, etc. Los patrones permiten la interpretación de regularidades presentes en diversas situaciones de la vida diaria por ejemplo en la música, en el movimiento, la economía, la geografía y la variación en general. El análisis cuidadoso de patrones y regularidades permite establecer generalizaciones. ( $p$. 51)

Es por esto que las progresiones aritméticas requieren del razonamiento inductivo para su acercamiento y comprensión, facilita la adquisición de conocimiento matemático como lo manifiestan Castro, et al. (2010). Por su parte, la resolución de problemas al desarrollar la comunicación favorece y se convierte en una herramienta para analizar el razonamiento de tipo inductivo a través de las explicaciones escritas y verbales que se plantea un estudiante al enfrentar un problema.

\section{Metodología}

El proceso de investigación llevado a cabo parte de la metodología cualitativa en tanto se pretende estudiar las explicaciones escritas y verbales a partir de patrones y regularidades que presentan los estudiantes del grado quinto de la Institución Educativa Bello Horizonte, al momento de resolver problemas relacionados con progresiones aritméticas en las sesiones de intervención; en ese sentido, se enmarca en las ideas que establecen Denzin y Lincoln (2005) cuando afirman que:

En este nivel la investigación cualitativa implica un acercamiento interpretativo y naturalista del mundo. Esto significa que los investigadores cualitativos estudian los objetos en sus escenarios naturales, intentando dar sentido, o interpretar los fenómenos en términos de los significados que las personas les dan. (p.5)

Es decir, el enfoque cualitativo sitúa al investigador en un contexto espacio-temporal, para lograr una interpretación del mundo o del fenómeno estudiado mediante instrumentos, en los cuales se consideraron tres aspectos que promueven las explicaciones escritas de los estudiantes; éstos fueron: completar, verificar y proponer. Además, la realización de entrevistas semiestructuradas para evidenciar las explicaciones verbales.

Ahora bien, la caracterización de la investigación cualitativa presentada, sugiere que ha de ser pertinente emplear el estudio intrínseco de casos como método de investigación el cual posibilita la comprensión de los casos particulares: estudiantes, en un contexto natural; al respecto, Stake (1999) menciona que el estudio de casos es el estudio de la particularidad y de la complejidad de un caso singular para llegar a comprender su actividad en circunstancias importantes, por ello se estudia un caso cuando se tiene un interés específico.

\section{Análisis de datos}

Se está llevando a cabo la aplicación de los instrumentos generados en el marco de la investigación para analizar las explicaciones escritas o verbales de los estudiantes. Dichos instrumentos contempla que el estudiante complete secuencias, verifique si la secuencias están bien elaboradas y proponga secuencias diferentes a las ya trabajadas explicando el por qué están "bien elaboradas". Finalizada la implementación de los instrumentos se procede con la triangulación de los datos cualitativos y con el análisis que permita obtener los resultados y las conclusiones, esperando que dicho proceso culmine para el mes de octubre del presente año.

\section{Conclusiones}

De acuerdo con lo anterior, esta investigación permite elaborar un instrumento que constituye una base para futuras investigaciones sobre las progresiones aritméticas en primaria, a la vez que posibilita generar un reconocimiento de que el razonamiento inductivo está involucrado y se debe fortalecer en las escuelas colombianas. Esta investigación, en sí, es un precedente del razonamiento inductivo a partir de las progresiones aritméticas. 


\section{Referencias}

Balacheff, N. (2000). Los Procesos de Prueba en los Alumnos de Matemáticas. Santa Fé de Bogotá: Una Empresa Docente.

Cañadas, M. C. (2007). Descripción y caracterización del razonamiento inductivo utilizado por estudiantes de educación secundaria al resolver tareas relacionadas con sucesiones lineales y cuadráticas. Universidad de Granada, Granada, España.

Denzin, N. K., \& Lincoln, Y. S. (2005). The Sage Handbook of Qualitative Research. Third Edition. Thousand Oaks: Sage Publications, Inc. Introduction. The Discipline and Practice of Qualitative Research. 1-13.

Castro, E., Cañadas, M. C., \& Molina, M. (2010). El razonamiento inductivo como generador de conocimiento matemático. UNO, 54, 55-67.

Gobernación de Antioquia. (2005). Interpretación e Implementación de los Estándares Básicos de Matemáticas. Recuperado de http://cmap.upb.edu. co/servlet/SBReadResource Servlet?rid $=1161187088328 \_488799458 \_19195$
Merino, E. (2012). Patrones y representaciones de alumnos de $5^{\circ}$ de educación primaria en una tarea generalización. Universidad de Granada, Granada, España.

Ministerio de Educación Nacional. (1998). Lineamientos Curriculares de Matemáticas. Recuperado de http://www.mineducacion. gov.co/cvn/1665/articles-89869_archivo_ pdf9.pdf

Ministerio de Educación Nacional. (2003). Estándares Básicos de Competencias en Matemáticas. Recuperado de http://www. mineducacion.gov.co/1621/articles-116042_ archivo_pdf2.pdf

Pólya, G. (1945). How to solve it. Princenton. University Press.

Pólya, G. (1966). Matemáticas y razonamiento plausible. Madrid: Tecnos

Stake, R. (1999). Investigación con estudios de casos. Madrid: Ediciones Morata, S. L. 